\title{
On producers of cosmic organic compounds: exploring the boron abundance in lithium-rich $\mathrm{K}$ giant stars
}

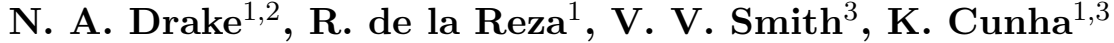 \\ ${ }^{1}$ Observatório Nacional-MCTIC, Rua Gen. José Cristino 77, 20921-400, Rio de Janeiro, Brazil, \\ email: drake@on.br, delareza@on.br, kcunha@on.br \\ ${ }^{2}$ St. Petersburg State University, Laboratory of Observational Astrophysics, \\ Universitetski pr. 28, 198504, St. Petersburg, Russia \\ ${ }^{3}$ National Optical Astronomy Observatory, 950 North Cherry Ave., Tucson, AZ 8 5719, USA, \\ email: vsmith@noao.edu, cunha@email.noao.edu
}

\begin{abstract}
The element boron belongs, together with lithium and beryllium, to a known trio of important elements for the study of evolutionary processes in low mass stars. Because B is the least fragile of this trio to be destroyed in the stellar interiors, it can be used to test if the $\mathrm{Li}$ enrichment is of planetary origin. Here, for the first time, boron lines are examined in the UV for four giants with different degrees of large Li enrichment by means of observations with the Hubble telescope. Two main results are found in our study. One is that to first approximation $\mathrm{B}$ abundances appear not to be in excess, invalidating the planet engulfment mechanism. The second one is that the two stars with very large Li abundances present emission lines indicating that quite strong active chromospheres are acting in these very Li-rich giants. These new results obtained from the UV complement our recent studies in the mid-IR (de la Reza et al. 2015) where strong emission-line features of organic material were found in the spectra of some Li-rich stars.
\end{abstract}

Keywords. stars: abundances - stars: chemically peculiar - stars: magnetic field - stars: mass loss - stars: individual: HD 787, HD 9746, HD 39853, HD 112127

\section{Introduction}

Following the standard evolution theory, the first ascent red giant stars (RGB) must destroy, by means of thermal reactions, almost all of their original lithium. However, since 1982, when Wallerstein \& Sneden detected the first K giant with strong lithium lines, HD 112127, several Li-rich K giants have been detected having Li abundances, even as large as ten times the original $\mathrm{Li}$ abundance, indicating that new sources of ${ }^{7} \mathrm{Li}$ are in action. As an example, Fig. 1 shows the spectrum of the Li-rich giant HD 19475 in the region containing the Li I resonance line at $\lambda 6707.8 \AA$.

$\mathrm{Li}, \mathrm{Be}$, and $\mathrm{B}$ are relatively fragile elements which are destroyed by $(p, \alpha)$ reactions at relatively low temperatures at about $2.5 \cdot 10^{6}, 3.5 \cdot 10^{6}$, and $5.0 \cdot 10^{6} \mathrm{~K}$, respectively. Since 1982 the nature of these Li-rich red giant stars have been involved in a problem known as the "lithium puzzle".

Recently we have discovered in the near-IR (de la Reza et al. 2015) the presence of complex organic compounds in the shells of Li-rich $\mathrm{K}$ giant stars (Fig. 2). These stars present large mass loss transforming them into sources of organic material in the Galaxy.

Beryllium abundances in lithium-rich giants, including the star HD 787, were studied by Castilho et al. (1999) and Melo et al. (2005). The authors have found that Be abundances in these stars are low, suggesting that Be is very depleted $(>90 \%)$ from the initial value of the Populaton I stars. 


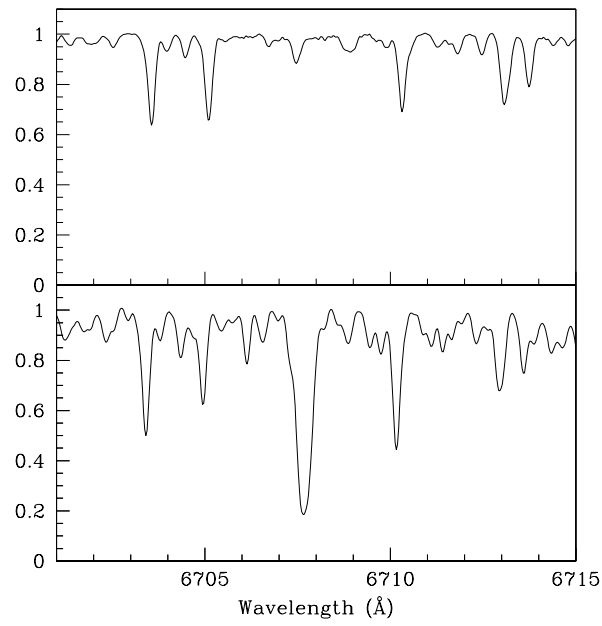

Figure 1. Spectrum of the Li-rich giant HD 787 (bottom panel) in comparison with a normal $\mathrm{K}$ giant star $\epsilon$ Vir (upper panel) in the region of the Li I line at $6707.8 \AA$.

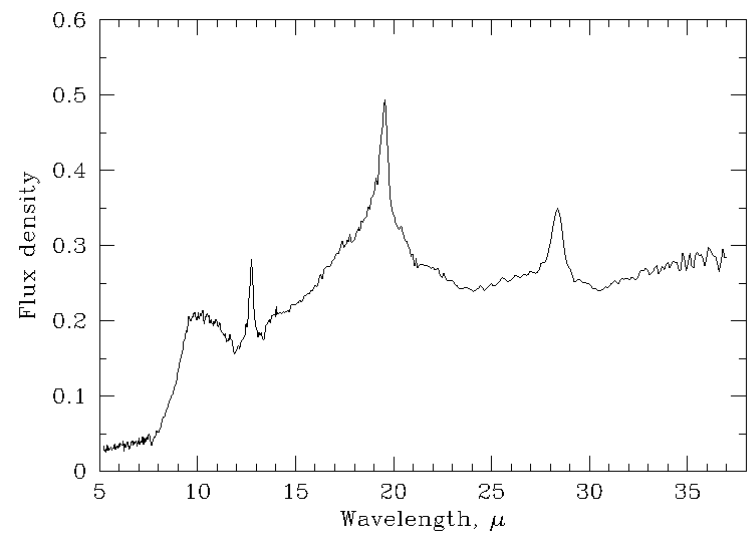

Figure 2. Spitzer mid-IR spectrum of the Li-rich K giant star PDS 68 between 5 and $38 \mu \mathrm{m}$ presenting the UIE features superposed on a strong continuum emission (de la Reza et al. 2015). The spectrum was taken from the Cornell Atlas of it Spitzer/IRS sources (CASSIS, Lebouteiller et al. 2011).

The nature of these stars is the object of recent studies. Here, it is explored for the first time the boron lines in the spectra of these objects in the UV spectral region with the aim to infer their main properties.

\section{Observations and stellar parameters}

We observed four Li-rich K giants HD 787, HD 9746, HD 3953, and HD 112127 with the Hubble Space Telescope and Space Telescope Imaging Spectrograph STIS with grating G230M having FWHM $=30 \mathrm{~km} \mathrm{~s}^{-1}$ and spectral region centered at the resonant B I lines at $\lambda 2496.8$ and $\lambda 2497.7 \AA$. These four spectra are shown in Fig. 3.

The atmospheric parameters of the stars, effective temperature $\left(T_{\text {eff }}\right)$, surface gravity 


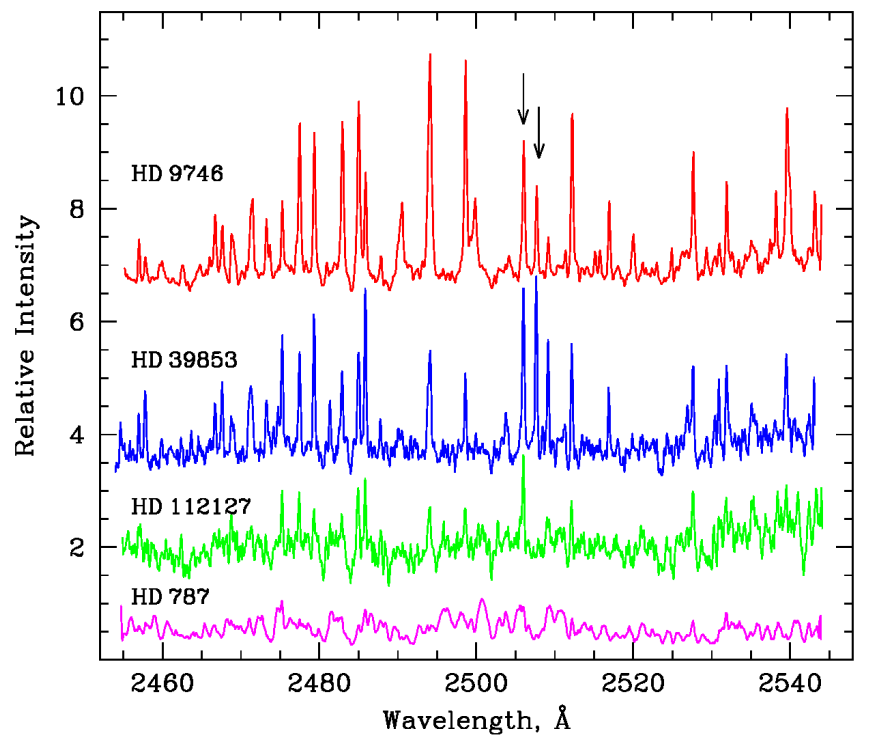

Figure 3. The HST/STIS UV spectra of four giant stars in the region of the B I lines at $\lambda 2496.8$ and $\lambda 2497.7 \AA$. From top to bottom: HD $9746(\log \varepsilon(\mathrm{Li})=3.75), \operatorname{HD} 39853(\log \varepsilon(\mathrm{Li})=2.85)$, HD $112127(\log \varepsilon(\mathrm{Li})=2.7)$, and HD $787(\log \varepsilon(\mathrm{Li})=1.8)$. The UV spectra of the Li-rich giants HD 9746 and HD 39853 are very complex and rich in strong emission lines, mainly of Fe II. As can be seen, the intensity of the emission lines correlates with the Li abundance. Very strong emission lines at $\lambda 2507$ and $\lambda 2509 \AA$ marked by arrows are observed in the spectra of HD 9746 and HD 39853. These two lines are the Fe II fluorescence lines pumped by H Ly $\alpha$ (Johanson \& Hamann 1993) pointing to the existence of very strong Ly $\alpha$ emission. Star HD 787 shows a different spectrum probably due to its binary nature. The spectra are arbitrary shifted in the relative intensity axis.

Table 1. Atmospheric parameters of observed Li-rich K giant stars.

\begin{tabular}{lcccc}
\hline Star & $T_{\text {eff }}, \mathrm{K}$ & $\log g$ & $\log \varepsilon(\mathrm{Li})$ & Reference \\
\hline HD 787* & 4220 & 1.50 & 1.8 & $(1)$ \\
HD 9746 & 4400 & 2.30 & 3.75 & $(2)$ \\
HD 39853 & 3900 & 1.16 & 2.85 & $(3)$ \\
HD 112127 & 4340 & 2.10 & 2.7 & $(4)$ \\
\hline
\end{tabular}

References: (1) Brown et al. (1989);

(2) Balachandran et al. (2000);

(3) Gratton \& D'Antona (1989);

(4) Wallerstein \& Sneden (1982)

Notes: ${ }^{*}$ The star HD 787 is a proper-motion binary

(Mason et al. 1999, Eggleton \& Tokovinin 2008)

$(\log g)$, and lithium abundance $(\log \varepsilon(\mathrm{Li}))\left(\right.$ where $\left.\log \varepsilon(\mathrm{X})=\log \left(N_{\mathrm{X}} / N_{\mathrm{H}}\right)+12.0\right)$, are shown in Table 1.

\section{Results and conclusions: how to explain the Li puzzle?}

- The boron abundances of the Li-rich giants appear to be low and in none of the cases presents an excess. This result is not compatible with any scenario of engulfment of planets in order to produce Li-rich K giant stars. 
- In de la Reza et al. (2015) we propose a qualitative scenario to try to explain the "Li puzzle" in K giant stars. Here, a mass mixing produced by the diminishing of the stellar core rotation in the RGB phase, reaches the surface layers of the stars forming eventually the observed circumstellar shell. At the same time, this mixing can, in some short time episodes, move the ${ }^{7}$ Be produced in the internal $\mathrm{H}$-burning region rapidly to the external convective zone where it is transformed into new ${ }^{7} \mathrm{Li}$.

- Privitera et al. (2016) suggested that a strong magnetic field in a red giant star may be a sign of a past planet engulfment. It is interesting to note that our observed very Li-rich K giant HD 9746 has an important magnetic field detected by Aurière et al. (2015). We found in this work that this $\mathrm{K}$ giant star can be a descendant of an Ap star on the main sequence known to have very high Li abundance. Because HD 9746 has a relatively high rotation of $v \sin i=8.7 \mathrm{~km} \mathrm{~s}^{-1}$,

its magnetic field can have two origins: one fossil from the Ap ancestor and one produced by a rotationally induced dynamo. Goriely (2007) suggested that high lithium abundance in magnetic Ap stars may be produced by the spallation reactions in the regions of the magnetic poles, where accelerated protons and $\alpha$-particles destroy CNO nuclei and produce lithium.

- Even if the direct relation of the Li enrichment phenomenon and existence of a magnetic field is not yet well understood, there exists a strong correlation between stellar activity and high Li abundances in red giant stars (Fekel \& Balachandran 1993, Drake et al. 2002).

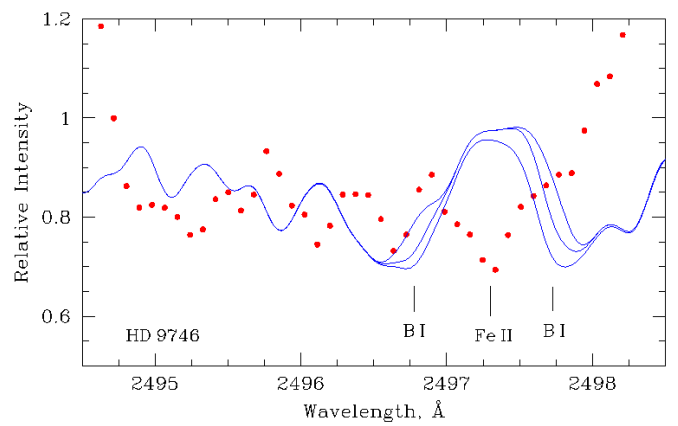

Figure 4. Fit of the observed spectrum of HD 9746 in the spectral region around the resonance doublet of B I at $\lambda 2496.8$ and $\lambda 2497.7 \AA$ in the spectrum of HD 9746. Points: observations. Lines: synthetic spectra computed using the recent version of MOOG code (Sneden 1973) for the boron abundances of $\log \varepsilon(\mathrm{B})=0.60,2.60$, and 4.60 corresponding to the $[\mathrm{B} / \mathrm{Fe}]=-2.0,0.0$, and +2.0. Strong contribution from the chromosphere causing veiling in this UV region, makes it difficult to determine the B abundance with high precision. Nevertheless, a direct comparison with theoretical B I lines shows that no expected B excess is present. The line lists were taken from the VALD Database, Duncan et al. (1998), and Cunha et al. (2000).

Acknowledgements. N.A.D. acknowledges IAU travel grant, FAPERJ, Rio de Janeiro, Brazil, for Visiting Researcher grant E-26/200.128/2015, and the Saint Petersburg State University for research grant 6.38.335.2015.

\section{References}

Aurière M., Konstantinova-Antova, R., Charbonnel, C., et al. 2015, A\& 4 , 574, A90

Balachandran S. C., Fekel, F. C., Henry, G. W., Uitenbroek, H. 2000, ApJ, 542, 978

Brown J. A., Sneden, C., Lambert, D. L., Dutchover, E. 1989, ApJS, 71, 293

Castilho, B. V., Spite, F., Barbuy, B. et al. 1999, A\&A, 345249 
Cunha K., Smith, V. V., Boesgaard, A. M., Lambert, D. L. 2000, ApJ, 530, 939

de la Reza R., Drake, N. A., Oliveira, I., Rengaswamy, S. 2015, ApJ, 806, 86

Drake N. A., de la Reza, R., da Silva, L., Lambert, D. L. 2002, AJ, 123, 2703

Duncan, D. K., Peterson, R. C., Thorburn, J. A., Pinsonneault, M. H. 1998, ApJ, 499, 871

Eggleton, P. \& Tokovinin, A. 2008, MNRAS, 389, 869

Fekel F. C. \& Balachandran S. 1993, ApJ, 403, 708

Goriely S. 2007, A\& A, 466, 619

Gratton R. G. \& D'Antona F. 1989, A\& A, 215, 66

Johansson S. \& Hamann F. 1993, Phys. Scr. T47, 157

Lebouteiller, V., Barry, D. J., Spoon, H. W. W., et al. 2011, ApJS, 196, 8

Mason, B. D., Martin, C., Hartkopf, W. I., et al. 1999, AJ, 117, 1890

Melo, C. H. F., de Laverny, P., Santos, N. C., et al. 2005, A\& A, 439, 227

Privitera, G., Meynet, G., Eggenberger, P., et al. 2016, A\& A, 593, L15

Sneden, C. 1973, PhD Thesis, Univ. of Texas at Austin

Wallerstein G. \& Sneden C. 1982, ApJ, 255, 577 